\title{
A TRANSCRIPTOMICALLY GUIDED COMPUTATIONAL QUANTIFICATION OF THE SRR8671434 RNA SEQUENCE
}

\author{
Rohan Bhansali \\ Loudoun Academy of Science \\ Ashburn, VA 20148 \\ rohanbhansali@connectai.org
}

February 2, 2021

\begin{abstract}
A BSTRACT
Ribonucleic acid (RNA) is a single strand nucleic acid responsible for genetic coding, decoding, regulation, and expression that consists of phosphate and ribose groups with several purposeful variants, notably messenger, transfer, and ribosomal RNA. RNA sequencing is a next-generation sequencing (NGS) technique capable of continuously analyzing cellular transcriptome and revealing the presence and quantity of RNA within a biological sample. The process entails reverse transcribing the extracted RNA into cDNA; subsequently, the cDNA is fragmented, enabling its input into an NGS workflow, after which adapters are added to both ends of the fragments. RNA sequencing is an expensive and time-consuming process due to its necessitation of an entire genomic library that is often difficult to analyze through traditional methods; however, applying computational methods can overcome these challenges through cutting edge data mining and informatics technologies. In this study, the SRR8671434 RNA sequence is quantified and analyzed for its ability to serve as an indicator for congenital afflictions. The findings in this paper can be applied towards preventing and curing associated diseases, as well as discerning other potential biomarkers within genetic materials.
\end{abstract}




\section{Introduction}

The purpose of this study is to quantitatively examine the SRR8671434 RNA sequence through data analysis and computational techniques [5].

\subsection{Ribonucleic Acid}

Ribonucleic acid (RNA) is a complex compound that replaces DNA as a genetic code carrier in cellular protein synthesis [3]. The structure of RNA was first described by R.W. Holley in 1965 and has since been the subject various studies exploring its function and evolution.

\subsubsection{Structure}

RNA is a single-stranded biopolymer made of a backbone consisting of alternating phosphate and ribose groups, each of which is attached to an adenine, uracil, cytosine, or guanine base [4]. Its three-dimensional structure allows the ribose and nitrogenous bases to be modified via cellular enzymes that attach chemical groups to the chain. These modifications lead to contortions within the RNA chain, further stabilizing its structure. RNAs are also capable of forming complexes with ribonucleoproteins (RNPs) which together serve as biological catalysts.

\subsubsection{Function}

Ribonucleic acid (RNA) is a single strand nucleic acid responsible for various biochemical tasks in coding, decoding, regulating, and expressing genes [1]. The central dogma of molecular biology suggest that the primary role of RNA is in converting DNA information to proteins by coding amino acids. RNA is also central in regulating cellular processes such as cell division, aging, and death.

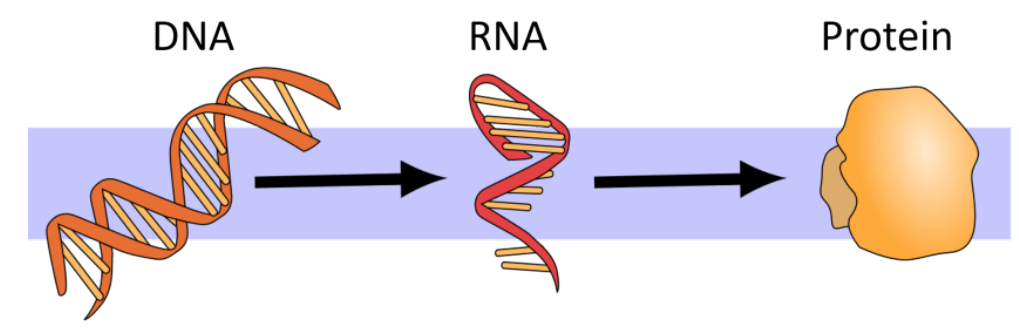

Figure 1: Central Dogma of Biology

\subsubsection{Types}

Although there are numerous types of RNA in both prokaryotic and eukaryotic cells, the most main types are messenger RNA (mRNA), transfer RNA (tRNA), and ribosomal RNA (rRNA) [5]. Although these types of RNAs are primarily responsible for carrying out biochemical reactions, they also can have complex regulatory functions in cellular processes and diseases.

mRNA carries genetic codes from DNA in the nucleus to ribosomes during protein synthesis as this is the location of protein translation in the cytoplasm [3]. rRNA encodes ribosome protein subunits which are synthesized in the nucleolus. Subsequently, they shift to the cytoplasm and read the mRNA code. The incorporation of specific amino acids in proteins is specified by the mRNA's sequence of three nitrogenous bases. The tRNA molecules transport the amino acids to the ribosomes, where they form proteins.

\subsubsection{Sequencing}

RNA sequencing is an emerging molecular technology that utilizes deep-sequencing techniques to examine the quantity and sequences of RNA in biological samples by analyzing the transcriptome of encoded gene expression patterns [2]. This technique is useful for detecting known or novel features within RNA sequences that can be linked to genetic diseases and disorders. 
Table 1: General Statistics

\begin{tabular}{lllllllll}
\hline Sample Name & $\%$ Assigned & M Assigned & $\%$ Dups & $\%$ GC & Length & $\%$ Failed & M Seqs & $\%$ Aligned \\
\hline SRR8671434 & $34.0 \%$ & 0.8 & $43.7 \%$ & $42 \%$ & $47 \mathrm{bp}$ & $30 \%$ & 2.1 & $69.3 \%$
\end{tabular}

\section{Analysis}

\subsection{General Statistics}

\section{2 featureCounts}

Subread featureCounts is a highly efficient general-purpose read summarization program that counts mapped reads for genomic features such as genes, exons, promoter, gene bodies, genomic bins and chromosomal locations.

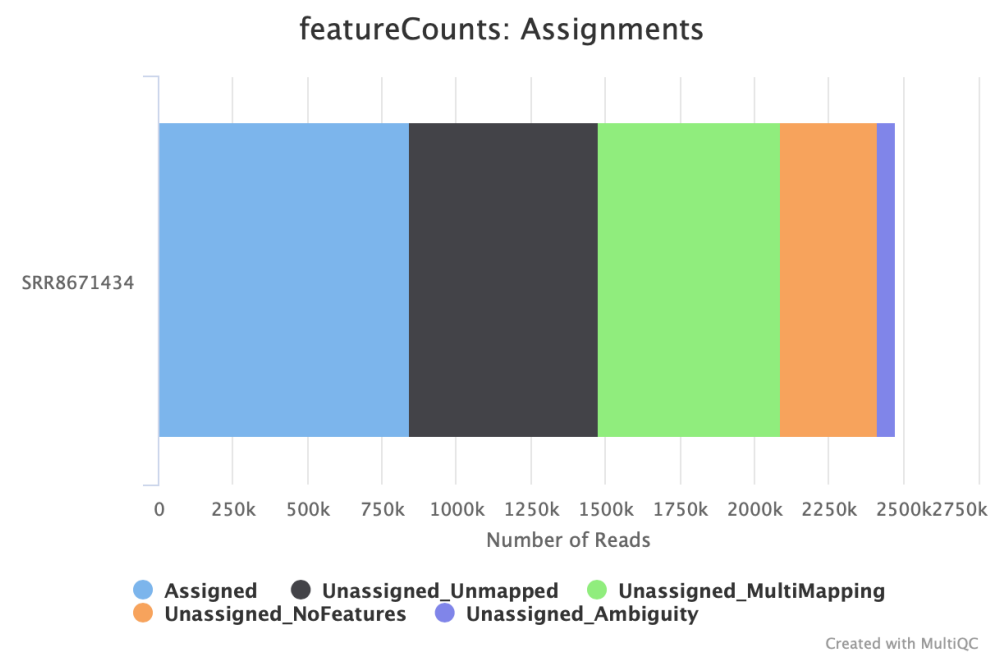

Figure 2: featureCounts: Assignments

\subsection{FastQC}

FastQC is a quality control tool for high throughput sequence data, written by Simon Andrews at the Babraham Institute in Cambridge.

\subsubsection{Sequence Counts}

Sequence counts for each sample. Duplicate read counts are an estimate only.

\subsubsection{Sequence Quality Histograms}

The mean quality value across each base position in the read.

\subsubsection{Per Sequence Quality Scores}

The number of reads with average quality scores. Shows if a subset of reads has poor quality.

\subsubsection{Per Base Sequence Content}

The proportion of each base position for which each of the four normal DNA bases has been called.

\subsubsection{Per Sequence GC Content}

The average GC content of reads. Normal random library typically have a roughly normal distribution of GC content. 


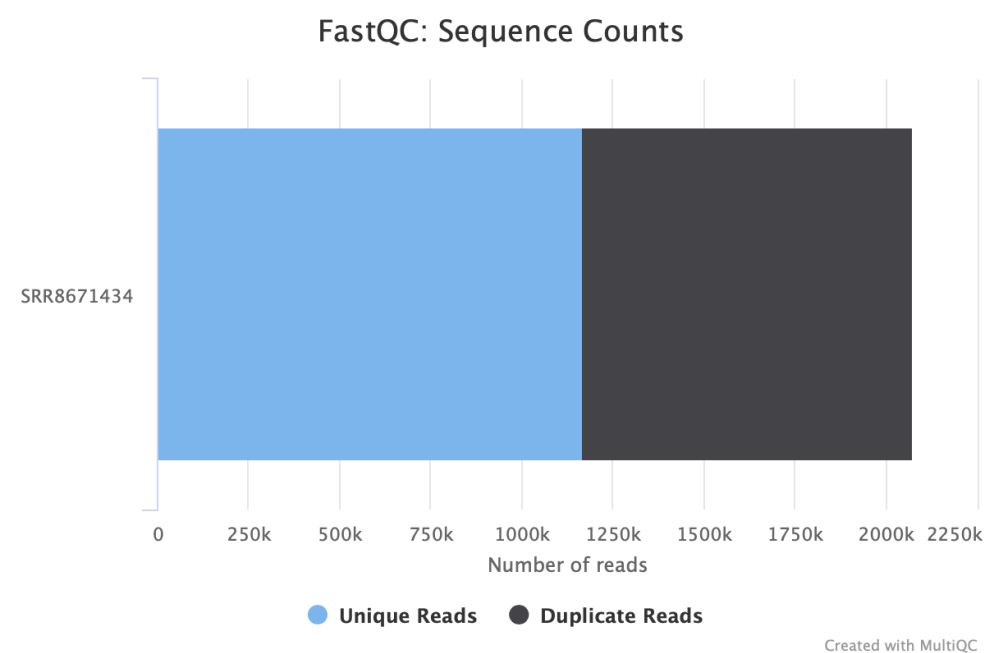

Figure 3: featureCounts: Sequence Counts

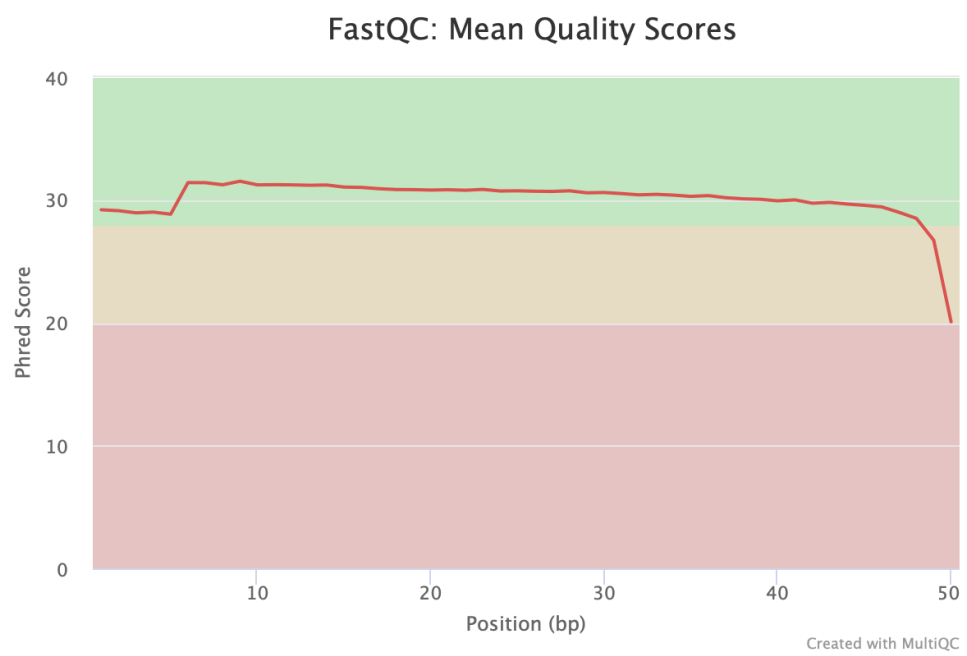

Figure 4: FastQC: Mean Quality Scores

\subsubsection{Per Base N Content}

The percentage of base calls at each position for which an $\mathrm{N}$ was called.

\subsubsection{Sequence Length Distribution}

The distribution of fragment sizes (read lengths) found.

\subsubsection{Sequence Duplication Levels}

The relative level of duplication found for every sequence.

\subsubsection{Overrepresented Sequences}

The total amount of overrepresented sequences found in each library; 1 samples had less than $1 \%$ of reads made up of overrepresented sequences. 
FastQC: Per Sequence Quality Scores

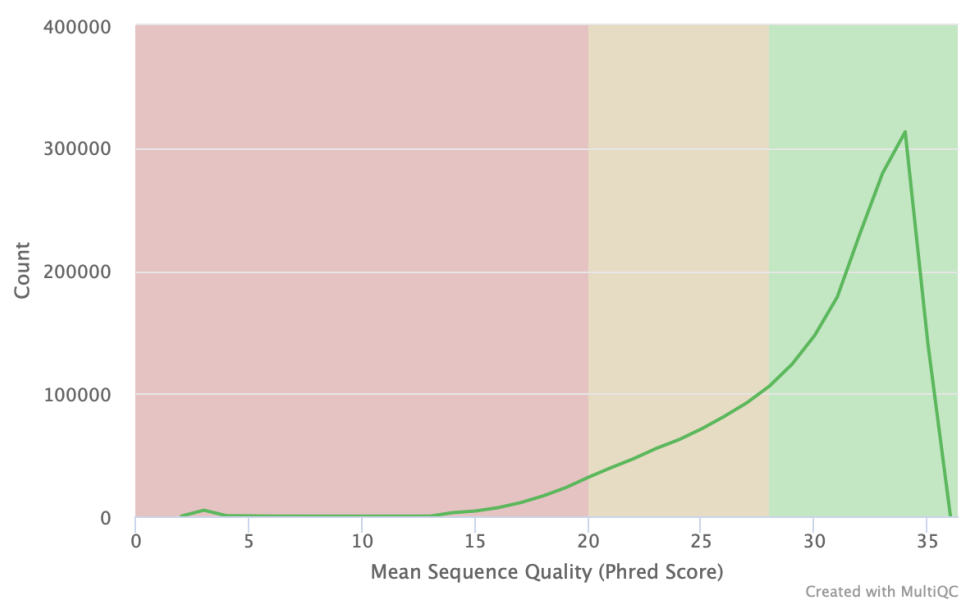

Figure 5: FastQC: Per Sequence Quality Scores

SRR8671434
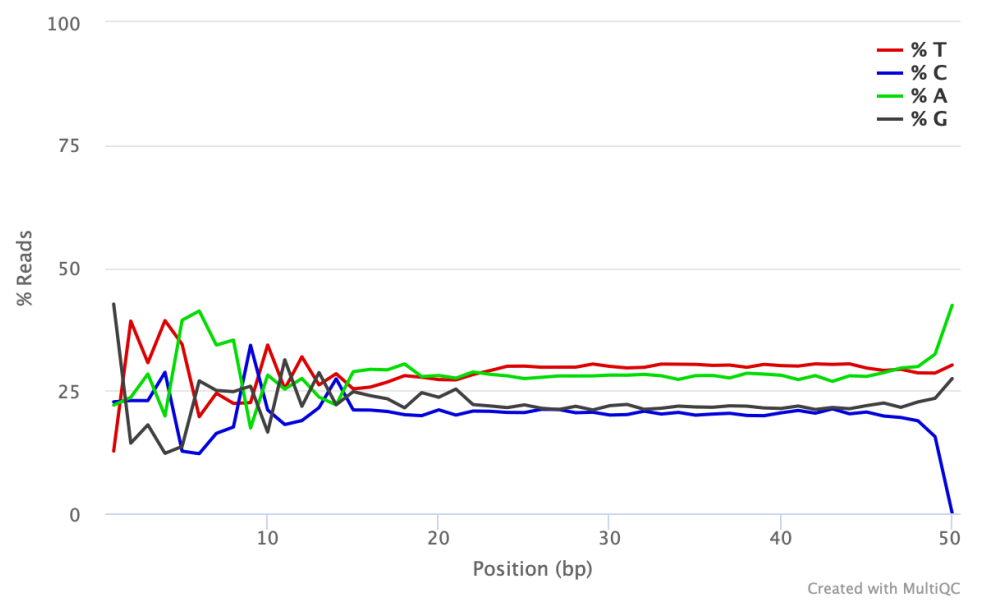

Figure 6: Per Base Sequence Content

\subsubsection{Adapter Content}

The cumulative percentage count of the proportion of the library which has seen each of the adapter sequences at each position; no samples found with any adapter contamination $>0.1 \%$.

\subsection{HISAT2}

HISAT2 is a fast and sensitive alignment program for mapping NGS reads (both DNA and RNA) against a reference genome or population of reference genomes.

\subsection{TPM}

\section{Conclusion}

From the results of this study, it can be concluded that the SRR8671434 RNA sequence holds significant promise in serving as a genetic predictor for various hereditary disease and carcinomas. The sequence points towards further research investigating its implications in transcriptomical technologies. Future studies should explore difference RNA sequences and assess their respective features and relations to different afflictions. 
Table 2: TPM

\begin{tabular}{l|llllll}
\hline Gene_Id & ExonLength & ExonReads & ExonTPM & IntronLength & IntronReads & IntronTPM \\
\hline ENSG00000248527 & 681.0 & 1844.0 & 3820.5 & 0.0 & 0.0 & 0.0 \\
ENSG00000240453 & 402.0 & 3.0 & 10.5 & 7202.0 & 0.0 & 0.0 \\
ENSG00000240409 & 207.0 & 43.0 & 293.1 & 0.0 & 0.0 & 0.0 \\
ENSG00000238009 & 3569.0 & 0.0 & 0.0 & 40703.0 & 1.0 & 0.3 \\
ENSG00000237973 & 1543.0 & 3102.0 & 2836.5 & 0.0 & 0.0 & 0.0 \\
ENSG00000237491 & 5104.0 & 0.0 & 0.0 & 26187.0 & 43.0 & 21.0 \\
ENSG00000237094 & 5950.0 & 1.0 & 0.2 & 130279.0 & 3.0 & 0.3 \\
ENSG00000230092 & 523.0 & 13.0 & 35.1 & 8760.0 & 0.0 & 0.0 \\
ENSG00000230021 & 1697.0 & 0.0 & 0.0 & 121418.0 & 8243.0 & 869.9 \\
ENSG00000229344 & 682.0 & 1625.0 & 3361.8 & 0.0 & 0.0 & 0.0 \\
ENSG00000228794 & 7631.0 & 0.0 & 0.0 & 24208.0 & 1.0 & 0.5 \\
ENSG00000227232 & 2073.0 & 152.0 & 103.5 & 13371.0 & 0.0 & 0.0 \\
ENSG00000225972 & 372.0 & 759.0 & 2878.7 & 0.0 & 0.0 & 0.0 \\
ENSG00000225630 & 1044.0 & 123.0 & 166.2 & 0.0 & 0.0 & 0.0 \\
ENSG00000223972 & 1756.0 & 43.0 & 34.5 & 788.0 & 0.0 & 0.0 \\
ENSG00000198744 & 547.0 & 893.0 & 2303.4 & 0.0 & 0.0 & 0.0 \\
ENSG00000188976 & 5539.0 & 100.0 & 25.5 & 9567.0 & 0.0 & 0.0 \\
ENSG00000187961 & 3395.0 & 5.0 & 2.1 & 1734.0 & 0.0 & 0.0 \\
ENSG00000187634 & 3205.0 & 89.0 & 39.2 & 16491.0 & 0.0 & 0.0 \\
ENSG00000187583 & 2833.0 & 1.0 & 0.5 & 6536.0 & 0.0 & 0.0 \\
\hline
\end{tabular}

Table 3: Unique TPM

\begin{tabular}{l|llllll}
\hline Gene_Id & ExonLength & ExonReads & ExonTPM & IntronLength & IntronReads & IntronTPM \\
\hline ENSG00000248527 & 0.0 & 0.0 & 0.0 & 0.0 & 0.0 & 0.0 \\
ENSG00000240453 & 9.0 & 3.0 & 679.5 & 7200.0 & 0.0 & 0.0 \\
ENSG00000240409 & 0.0 & 0.0 & 0.0 & 0.0 & 0.0 & 0.0 \\
ENSG00000238009 & 1821.0 & 0.0 & 0.0 & 38354.0 & 1.0 & 1.9 \\
ENSG00000237973 & 0.0 & 0.0 & 0.0 & 0.0 & 0.0 & 0.0 \\
ENSG00000237491 & 3172.0 & 0.0 & 0.0 & 18937.0 & 30.0 & 114.1 \\
ENSG00000237094 & 4272.0 & 1.0 & 0.5 & 125189.0 & 3.0 & 1.7 \\
ENSG00000230092 & 42.0 & 2.0 & 97.1 & 6.0 & 0.0 & 0.0 \\
ENSG00000230021 & 1504.0 & 0.0 & 0.0 & 111070.0 & 1014.0 & 657.3 \\
ENSG00000229344 & 0.0 & 0.0 & 0.0 & 0.0 & 0.0 & 0.0 \\
ENSG00000228794 & 7631.0 & 0.0 & 0.0 & 24208.0 & 1.0 & 3.0 \\
ENSG00000227232 & 1770.0 & 127.0 & 146.3 & 13371.0 & 0.0 & 0.0 \\
ENSG00000225972 & 0.0 & 0.0 & 0.0 & 0.0 & 0.0 & 0.0 \\
ENSG00000225630 & 0.0 & 0.0 & 0.0 & 0.0 & 0.0 & 0.0 \\
ENSG00000223972 & 1706.0 & 0.0 & 0.0 & 788.0 & 0.0 & 0.0 \\
ENSG00000198744 & 0.0 & 0.0 & 0.0 & 0.0 & 0.0 & 0.0 \\
ENSG00000188976 & 5167.0 & 17.0 & 6.7 & 9567.0 & 0.0 & 0.0 \\
ENSG00000187961 & 3395.0 & 5.0 & 3.0 & 1734.0 & 0.0 & 0.0 \\
ENSG00000187634 & 2532.0 & 0.0 & 0.0 & 11610.0 & 0.0 & 0.0 \\
ENSG00000187583 & 2679.0 & 1.0 & 0.0 & 6023.0 & 0.0 & 0.0 \\
\hline
\end{tabular}


FastQC: Per Sequence GC Content

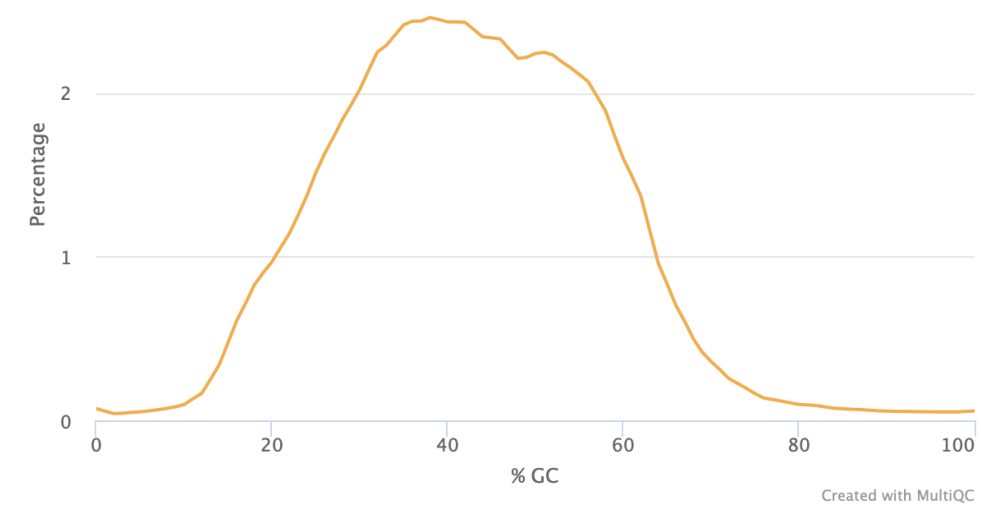

Figure 7: FastQC: Per Sequence GC Content

FastQC: Per Base N Content

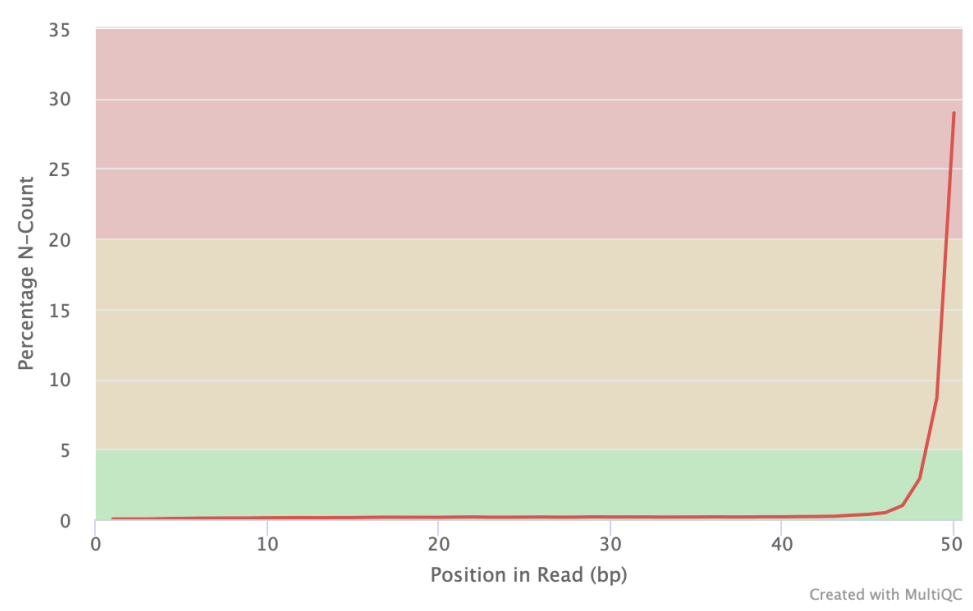

Figure 8: FastQC: Per Base N Content

\section{References}

[1] Conesa, A., Madrigal, P., \& Tarazona, S. (2016). A survey of best practices for RNA-seq data analysis. Genome Biology, 17(13). doi:10.1007/s00018-009-0180-6

[2] Haas, B. \& Zody, M. (2010). Advancing RNA-Seq analysis. Nature Biotechnology, 28 421-423. doi:10.1038/nbt0510-421

[3] Marguerat, S. \& Bähler, J. (2010). RNA-seq: from technology to biology. Cellular and Molecular Life Sciences, 67 569-579. doi:10.1007/s00018-009-0180-6

[4] Pepke, S., Wold, B., \& Mortazavi, A. (2010). Computation for ChIP-seq and RNA-seq studies. Nature Methods, 6 S22-S32. doi:10.1038/nmeth.1371

[5] Wang, Z., Gerstein, M., \& Snyder, M. (2009). RNA-Seq: a revolutionary tool for transcriptomics. Nature Reviews Genetics, 10 57-63. doi:10.1038/nrg2484 
FastQC: Sequence Length Distribution

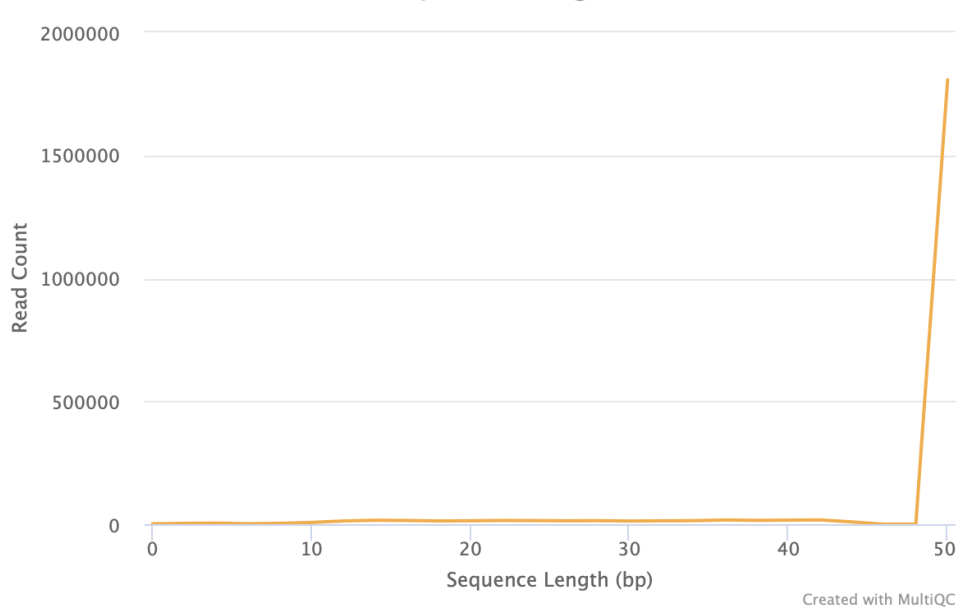

Figure 9: FastQC: Sequence Length Distribution

FastQC: Sequence Duplication Levels

100

75

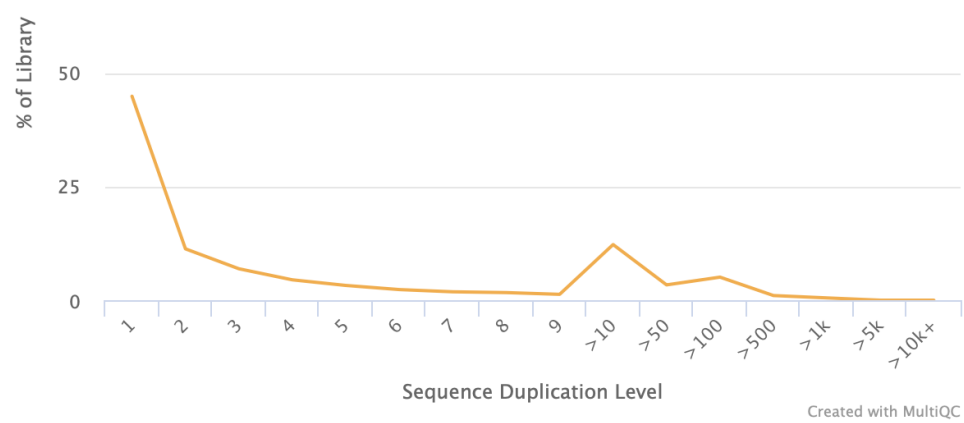

Figure 10: FastQC: Sequence Duplication Levels

HISAT2: SE Alignment Scores

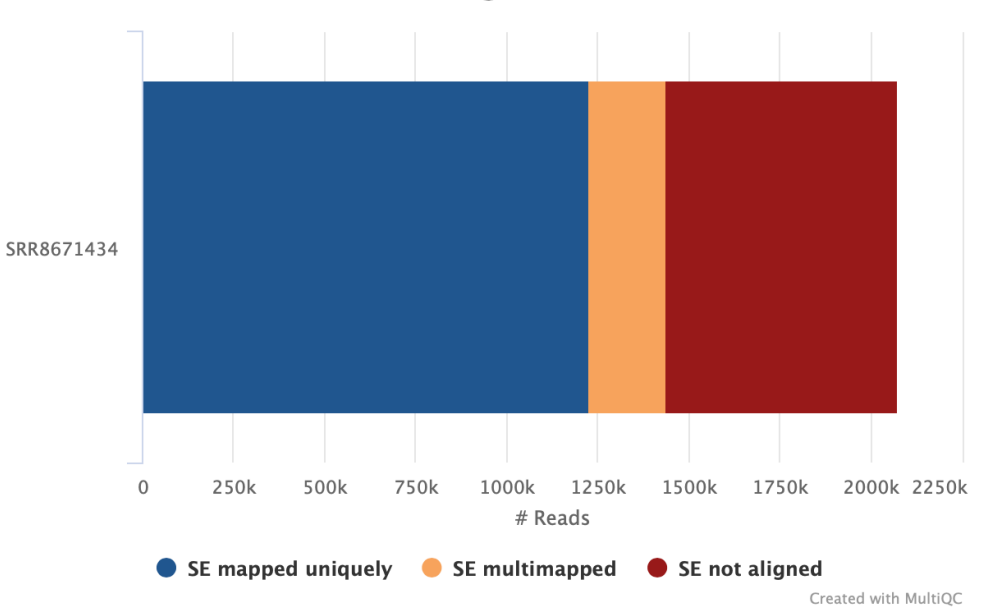

Figure 11: HISAT2: SE Alignment Scores 